SCIO. Revista de Filosofia, n. ${ }^{\circ}$ 19, Noviembre de 2020, 229-259, ISSN: 1887-9853

\title{
LA ENSEÑANZA EN LÍNEA Y LA DIMENSIÓN ARTIFICIAL DE LA CIENCIA DE LA EDUCACIÓN: ANÁLISIS FILOSÓFICO-METODOLÓGICO COMO CIENCIA DE DISEÑO
}

\author{
ONLINE TEACHING AND THE ARTIFICIAL DIMENSION \\ OF SCIENCE OF EDUCATION:
}

A PHILOSOPHICAL-METHODOLOGICAL ANALYSIS AS A SCIENCE OF DESIGN

Ana María Alonso Rodríguez $a^{a^{*}}$

Fechas de recepción y aceptación: 9 de junio de 2020 y 30 de julio de 2020

DOI: $10.46583 /$ scio_2020.19.687

Resumen: La educación en línea plantea nuevos problemas a la ciencia de la educación, que tiene un carácter dual: es ciencia social y ciencia de lo artificial. La mediación de la tecnología refuerza la dimensión artificial de la ciencia de la educación, que se configura como una ciencia de diseño. Esto revierte en la vertiente interna y la dimensión externa de la actividad educativa.

Este artículo ofrece un análisis filosófico-metodológico de la educación mediada por la tecnología. Lo hace desde el marco teórico de las ciencias de lo artificial, lo que aporta novedad: por un lado, este enfoque es poco habitual; y, por otro, la filosofía de la ciencia apenas ha prestado atención a la ciencia de la educación.

Dentro de este marco temático, para resolver problemas en los procesos de enseñanza mediados por la tecnología, se contextualizan los diseños educativos como diseños científicos. Después se analizan sus límites para atender las distintas dimensiones de la educación

\footnotetext{
${ }^{a}$ Centro de Investigación de Filosofía de la Ciencia y la Tecnología (CIFCYT). Facultad de Humanidades y Documentación. Universidad de A Coruña

${ }^{*}$ Correspondencia: Centro de Investigación de Filosofía de la Ciencia y la Tecnología (CIFCYT). Calle Dr. Cabrera s/n. 15403 (Ferrol), La Coruña. España.

E-mail: a.alonsor@udc.es
} 
Palabras clave: ciencia de la educación, ciencias de lo artificial, educación mediada por la tecnología, tecnología educativa, enseñanza on-line, diseño pedagógico.

Abstract: Online education poses new problems for education science, which has a dual character: it is both a social science and a science of the artificial. The mediation of technology reinforces the artificial dimension of education science, which is configured as a design science. This affects the internal aspect and the external dimension of the educational activity.

This paper offers a philosophical-methodological analysis of technology-mediated education, from the theoretical framework of the artificial sciences. It does so from the theoretical framework of the artificial sciences, which can be innovative: on the one hand, it is an unusual approach; on the other hand, the philosophy of science has scarcely paid any attention to the science of education.

Within this thematic framework, educational designs are contextualized as scientific designs to solve problems in teaching processes mediated by technology. Then, its limits are analysed to tackle the different dimensions of education.

Keywords: science of education, sciences of the artificial, technology-mediated education, educational technology, on-line teaching, pedagogical design.

\section{$\S 1$. NueVo ESCENARIO PARA LA EDUCACIÓN}

Desde el punto de vista filosófico-metodológico, la enseñanza en línea (online) plantea un reto. Para satisfacer las nuevas demandas sociales de aprendizaje permanente, la tecnología de la información y la comunicación ha impulsado un rápido desarrollo de esta modalidad educativa. Tanto por su difusión como por su actual relevancia, plantea cuestiones filosófico-metodológicas de indudable calado. Esto no se apreció en los primeros momentos de su implantación, ya que la enseñanza en línea se planteó inicialmente como una opción complementaria. Estaba pensada para la ampliación de la cobertura educativa, principalmente para responder a las demandas de entornos de aprendizaje 
flexibles. Posteriormente fue ampliando su radio de acción para cubrir las crecientes necesidades de la educación superior.

Su carácter innovador, en lo que atañe a los recursos tecnológicos utilizados y la creatividad que aporta la metodología del diseño, ha situado la enseñanza en línea en la vanguardia educativa. Ha mostrado una gran capacidad para integrar distintas estrategias y recursos al servicio de las necesidades de aprendizaje y, sobre todo, para adaptarse a un entorno cambiante. Pero su implementación en el ámbito de la educación formal siempre se ha considerado con cautela, por tratarse de un proceso complejo. Además, suscita no pocas resistencias, tanto en los docentes como en las entidades educativas.

Sin embargo, la situación de excepcionalidad provocada por la COVID-19, ha permitido ver la enseñanza en línea como una alternativa provisionalmente viable del sistema educativo. Ha puesto de manifiesto algunas disfunciones estructurales para el desarrollo de la actividad educativa ${ }^{1}$. A su vez, con respecto a la educación convencional, ha mostrado algunas de sus ventajas y limitaciones. Ha permitido además contrastar su aceptación social. En cualquier caso, se ha acelerado la transición a una nueva etapa educativa, donde prevalecen las pantallas frente a la interacción cara a cara del enfoque educativo presencial. Esto obliga a pensar filosóficamente la ciencia de la educación desde un nuevo ángulo, para abordar la novedad que supone la enseñanza en línea.

\subsection{Rasgos característicos de la enseñanza en línea}

Los rasgos característicos de la enseñanza en línea, por los que difiere de la enseñanza convencional, se concretan en diversos factores: (i) la distancia transaccional en las relaciones educativas; (ii) una mayor flexibilidad, que redunda en la autonomía de los estudiantes para desarrollar y mantener

\footnotetext{
${ }^{1}$ Parte de las disfunciones se deben a dificultades del entorno, ya que los modelos de organización siguen siendo los propios de la educación convencional. En ese contexto, hubo que improvisar y aplicar precipitadamente los diseños educativos. En consonancia con las competencias del profesorado y el alumnado, estos diseños muchas veces reproducen metodologías y estrategias didácticas que no aprovechan el potencial de la tecnología. Todavía hay que contar, entre las dificultades, también las referidas al software disponible o la falta de conectividad en determinados territorios.
}

SCIO. Revista de Filosofí, n. ${ }^{\circ}$ 19, Noviembre de 2020, 229-259, ISSN: 1887-9853 
el control sobre el proceso de aprendizaje ${ }^{2}$; y también (iii) formas distintas de interacción en diferentes ámbitos: alumno-contenido, alumno-instructor, alumno-alumno, alumno-interfaz (Gunawardena y McIsaac, 1996).

En ello incide que, en su configuración, la enseñanza en línea depende de la mediación de la tecnología: $a$ ) las TIC, conectadas a internet, ponen a disposición de docentes y estudiantes un gran volumen de información y lo hacen de forma inmediata; $b$ ) mediante las TIC enlazadas a la red se puede trabajar de manera conjunta desde lugares distintos; $c$ ) a través de la red están disponibles una gran diversidad de recursos didácticos; $d$ ) este soporte y los contenidos accesibles en la web o en la nube facilitan la colaboración de expertos y un acercamiento interdisciplinar a los problemas científicos (Childers y Gail Jones, 2015).

Ahora bien, la verdadera novedad de la mediación tecnológica para la enseñanza en línea se encuentra en otro rasgo: atañe al propio aprendizaje. El aprendizaje en línea es distinto al que se adquiere en la educación presencial, puesto que la tecnología, como dice Laurillard (2012: 3) "da forma a lo que se aprende al cambiar cómo es aprendido". Así, la cuestión metodológica clave no es ahora la que afecta al conocimiento ya articulado. Es otra la incidencia metológica, pues es previa e incide en la propia construcción del conocimiento. Más aún, tiene mayor repercusión epistemológica, puesto que afecta incluso a las habilidades necesarias para el acceso a la información.

\subsection{Reforzamiento de la componente artificial en la tarea educativa}

Mediante este giro epistemológico y metodológico -al variar las formas de acceso, de generación y difusión del conocimiento científico- las tecnologías han creado un nuevo escenario. Aunque inicialmente extrínseco, está siendo internalizado, puesto que está modificando también los modos de hacer ciencia y, en particular, la ciencia de la educación ${ }^{3}$. La clave filosófico-metodoló-

${ }^{2}$ Se traslada así el foco de atención de la educación de la enseñanza al aprendizaje.

${ }^{3}$ La informatización y la telematización han revolucionado las prácticas científicas. En el caso de la Educación, el modelo en red de propagación de la información que instauró internet aporta novedades. Tiene ventajas con respecto a los modos tradicionales de producción de conocimiento en educación. 
gica es que inciden directamente en su componente artificial y la refuerzan. Esto afecta tanto a nivel teórico (al pensar en objetivos, procesos y resultados) como en el plano de la práctica, con formas nuevas de hacer las cosas en las organizaciones educativas.

En consecuencia, una reflexión acerca de la educación que abarque la enseñanza en línea supone reconocer una componente artificial en la tarea educativa. Está dimensionada de modo especial por el soporte tecnológico disponible. Esto conduce a concebir la educación como ciencia aplicada de diseño, junto con su consideración como ciencia social. La presencia de internet, además de aportar la faceta tecnológica de las tecnologías de la información y la comunicación (TIC) y la dimensión social de la red, involucra también a las ciencias de internet (González, 2018). Esto configura un campo de investigación que se encuentra en la interfaz entre dos áreas disciplinarias altamente complejas (Laurillard, 2007: 357).

Sobre esta base, el carácter dual (social y artificial) de la ciencia de la educación se refuerza en la enseñanza en línea. Aunque el objeto de estudio sigue siendo la actividad educativa, los problemas que se abordan desde ambas perspectivas son distintos. Son también diferentes los métodos que emplean $\mathrm{y}$, por tanto, los resultados que obtienen, que generan consecuencias para los diversos agentes educativos. A este respecto, a diferencia de las ciencias sociales que son preferentemente analíticas, las ciencias de lo artificial buscan principalmente sintetizar (Simon, 1996: 4).

Desde el punto de vista filosófico-metodológico, cabe considerar la enseñanza en línea en este marco de ciencias aplicadas, que buscan hacer del mundo un lugar mejor al añadir nuevas posibilidades. Desde un punto de vista interno, usan el conocimiento para mejorar las cosas, acercándose a como "deberían ser". Para ello, combinan la predicción y la prescripción (González, 2015). Desde la perspectiva externa, mejorar las cosas en educación, atañe a los aprendizajes de los estudiantes, en el actual contexto de la sociedad del conocimiento. Esto supone un cambio dentro de un entorno donde el conocimiento prevalece sobre el medio físico o el tradicional ámbito social.

Para el desarrollo de la enseñanza en línea, hay apoyo en la creatividad científica y la innovación tecnológica. Internet proporciona una base para resolver los problemas concretos de otra forma, distinta a la tradicional. A través 
de la mediación de la red de redes, cabe formular objetivos más ambiciosos, planificar procesos usando formas organizativas novedosas - una ruta horizontal de difundir la información- con la intención de obtener mejores resultados en el aprendizaje.

Desde la perspectiva externa, el condicionante social de tipo físico se diluye: cabe conectar con puntos muy distantes del foco educativo. También aquí se aprecia la componente artificial de la ciencia de la educación, que dilata los procesos de enseñanza-aprendizaje en nuevas direcciones. Se sitúa en una sociedad interconectada y potencialmente global, aunque los niveles formativos tengan relación con las respectivas edades y tipo de conocimiento disponible en los usuarios de la enseñanza en línea.

Considero que el gran cambio filosófico-metodológico de la enseñanza en línea consiste en la "cientificación" (Niiniluoto, 1993: 11) efectiva de los diseños pedagógicos. En este sentido, primero la vertiente científica de internet -que se basa en diseños que son, con frecuencia, de inteligencia artificial-y, después, la faceta tecnológica de la red propician la aceleración del proceso de cientificación que ha experimentado la ciencia de la educación ${ }^{4}$. Así, es cada vez más una ciencia de diseño y, a este respecto, también ha aumentado su dependencia con los desarrollos de la inteligencia artificial ${ }^{5}$. Esto, a su vez, provoca el riesgo de reforzar el enfoque de "inteligencia computacional" (González, 2017), que es dominante en esa modalidad educativa.

\section{§2. LA CIENCIA DE LA EDUCACIÓN EN LA SOCIEDAD DEL CONOCIMIENTO}

La enseñanza en línea se sitúa dentro de la sociedad del conocimiento. A este respecto, junto a la vertiente científica de la educación en línea está la faceta tecnológica, que ha introducido cambios significativos. La tecnología no es mero soporte, puesto que es también un condicionante destacado de la

\footnotetext{
${ }^{4}$ A mi juicio, lo que ha impulsado avances significativos en la dimensión artificial de la ciencia de la educación ha sido su implicación en la búsqueda de usos efectivos de la tecnología, para optimizar los procesos de enseñanza-aprendizaje.

${ }^{5}$ Las máquinas están aprendiendo sobre las personas, a partir de los datos que ellas generan en el proceso de aprendizaje.
} 
actividad educativa en línea. Así, afecta a los elementos centrales de la propia actividad educativa: $a$ ) los modelos de enseñanza; $b$ ) los tipos de aprendizaje; c) las fuentes de información y conocimiento, que ahora son multirreferenciales; d) los procesos cognitivos; etc.

Por un lado, esto provoca la desestabilización de los marcos filosóficometodológicos e institucionales en los que se venía desarrollando la acción educativa; $y$, por otro lado, cuestiona los modelos de enseñanza y aprendizaje que eran generalmente aceptados ${ }^{6}$. De ahí que la ciencia de la educación necesite marcos conceptuales más amplios y profundos, para comprender y utilizar la dimensión educativa y formativa de las tecnologías?

La incorporación de internet ha propiciado un conjunto amplio de posibilidades para la educación, que conciernen a las tres vertientes de la red de redes: la científica, la tecnológica y la social (González, 2018). En cada una de ellas ha dejado su huella, aunque la más resaltada ha sido la tercera, relacionada con la sociedad del conocimiento, cuyo desarrollo requiere un sistema diseñado para optimizar el aprendizaje $\mathrm{e}^{8}$.

Con la red de redes, se han ampliado las modalidades en las que se realiza el aprendizaje: $(i)$ se mantiene la modalidad presencial, que presenta algunas novedades, (ii) se ha desarrollado una modalidad mixta, presencial y no-presencial (blended learning), y (iii) se incorpora una nueva forma de aprendizaje directamente mediado por la tecnología, que es netamente electrónico (e-learning).

${ }^{6}$ A esto hay que añadir también cambios institucionales y organizativos en los diversos niveles de la enseñanza.

${ }^{7}$ Ya en las últimas décadas del siglo xx, se impusieron áreas novedosas de interés prioritario en la investigación educativa: nuevos entornos de aprendizaje, nuevas formas de interacción educativa, alfabetización digital, etc., que dieron lugar a una amplia variedad de estudios.

${ }^{8}$ Una de las pocas cosas sobre las cuales prácticamente todos los autores están de acuerdo es que las personas tienen necesidades de aprendizaje diferentes y aprenden a ritmos distintos. Sin embargo, las escuelas y los programas de formación en general enseñan una cantidad fija y predeterminada de contenidos, en un cierto intervalo de tiempo preestablecido. Planificar el desarrollo de la enseñanza, de modo que cada estudiante avance en el aprendizaje al ritmo en el que va alcanzando un determinado nivel de logro, requiere la aplicación de una metodología didáctica que internet permite optimizar.

SCIO. Revista de Filosofía, n. ${ }^{\circ}$ 19, Noviembre de 2020, 229-259, ISSN: 1887-9853 
(i) La educación presencial continúa desarrollándose en escenarios tradicionales como el aula ${ }^{9}$, donde se usan medios y recursos en soportes convencionales. Aunque las TIC tienen ahora su espacio físico en estos escenarios, su uso está destinado básicamente al aprovechamiento de materiales diseñados de forma previa. Esto no modifica sustancialmente los procesos educativos (aunque no puede afirmarse que no los modifique en absoluto).

(ii) En la modalidad mixta de aprendizaje (blended learning, b-Learning), el proceso educativo combina e integra medios tecnológicos y medios convencionales. Esto sucede con actividades que se realizan en el aula, de forma presencial, y con la orientación de un profesor (es decir, de un "tutor humano"). Aquí el uso de las TIC no solo complementa, sino que propicia una mayor variedad de métodos, experiencias y dinámicas de aprendizaje ${ }^{10}$.

(iii) Aunque el aprendizaje a distancia no es novedoso, sí lo es, en términos de procesos educativos, el aprendizaje electrónico (completamente mediado por las TIC) o e-learning. En esta modalidad, el proceso de enseñanza-aprendizaje se realiza a través de la red de redes ${ }^{11}$. Ahí la interacción del estudiante con el material está mediada por herramientas informáticas.

Ciertamente la presencia de internet en la enseñanza refuerza el carácter artificial de la ciencia de la educación. Ya sea con su presencia en el aula a través de las pantallas de las TIC, con la modalidad combinada o mediante el aprendizaje electrónico en línea, parece claro que se trabaja a partir de diseños, donde destacan los elementos de índole artificial: a) Aparecen en los objetivos buscados, que pueden ser completamente novedosos. Son se-

\footnotetext{
${ }^{9}$ También lo hace en otros espacios, tales como la biblioteca o los laboratorios en el centro educativo, los museos, las excavaciones arqueológicas, etc. Respecto s un hipotético viajero del tiempo del siglo XIX, es habitual señalar que desconocería y se sorprendería al visitar un quirófano, una fábrica o, incluso, un banco transformados por la tecnología. Sin embargo, reconocería el espacio del aula y las actividades que en ellas se llevan a cabo, ya que apenas han cambiado. A mi juicio, esto no es del todo correcto.

${ }^{10}$ La enseñanza y el aprendizaje que se realizan en un medio tecnológico son asincrónicos, mientras que el presencial es sincrónico, lo que obliga a transitar de uno a otro.

${ }^{11}$ Es preciso reparar en que la red de redes tiene una estructura por capas (layers), donde hay que distinguir el plano de la infraestructura tecnológica (internet, en sentido estricto), el nivel de la web y el lugar de la nube y las aplicaciones prácticas conocidas como apps (González, 2019).
} 
leccionados de forma deliberada, de acuerdo con criterios que con frecuencia son de carácter práctico. b) Los procesos se llevan a cabo en una plataforma artificial, que requiere el dominio de unas destrezas aprendidas meramente instrumentales (otra forma de lo artificial). c) Los resultados se miden principalmente en relación con aspectos cognitivos y operativos. Sin embargo, retroalimentan el proceso educativo y lo llevan hacia metas que pueden ser bastante diferentes de las propias de la enseñanza tradicional.

Mediante esta vinculación entre la ciencia de la educación e internet, se han ampliando las oportunidades educativas. Aunque el resultado es difícil de anticipar, se espera que permita mejorar las estrategias de enseñanza con el fin de optimizar el aprendizaje. Esta relación es el objeto de indagación de la "tecnología educativa" (TE), un ámbito disciplinar que se ocupa de los procesos de enseñanza mediados por la tecnología en diferentes entornos educativos. A través de diferentes conceptualizaciones, su campo de estudio no se limita, en la actualidad, a la incorporación de los medios y recursos tecnológicos a las situaciones de enseñanza. La tecnología educativa busca la aplicación del conocimiento científico y la creación de diseños para resolver problemas concretos en contextos educativos específicos (Cabero, 2001).

Sucede que la educación mediada por la tecnología tiene cada vez más una mayor incidencia en el resto de las actividades humanas. Pero, hasta la fecha, no parece haber centrado la preocupación de los filósofos de la ciencia. Esto se engloba en una cuestión más amplia, pues no existe, en rigor, una filosofía y metodología especial de la educación que haya alcanzado un nivel de desarrollo comparable a otras disciplinas (como sucede con la filosofía de la física, la filosofía de la biología o la filosofía de la economía). A este respecto, el análisis de los especialistas en el tema educativo necesita completarse con la visión más abarcante que aporta la filosofía y metodología de la ciencia.

Así, aun cuando la consideración de la ciencia de la educación como una ciencia de diseño no es desconocida, se echa de menos su fundamentación filosófico-metodológica en cuanto que ciencia de lo artificial. Orientar ese análisis es el objetivo central de este artículo.

Pero procede matizar que la educación mediada por la tecnología constituye un ámbito de investigación interdisciplinar en el que, además de la ciencia de la educación, interviene internet (en su vertiente científica y su 
faceta tecnológica). Cuenta además con desarrollos de la inteligencia artificial, dentro de la sociedad del conocimiento. Todo ello configura un escenario novedoso:

1. La educación y la tecnología pueden interactuar de muchas maneras, a través de la interrelación entre la creatividad científica y la innovación tecnológica.

2. La incorporación de la tecnología en la actividad educativa puede tener influencias epistemológicas, metodológicas y ontológicas, que podrían incidir en la configuración de la inteligencia humana.

3. La ciencia de la educación y las ciencias de internet: web science, network science, etc. (Tiropanis, Hall, Crowcroft, Contractor y Tassiulas, 2015), así como también la inteligencia artificial, se ubican en el ámbito temático de las ciencias de lo artificial. Todas ellas trabajan con diseños que buscan objetivos específicos, siguiendo procesos seleccionados para lograr los resultados esperados.

4. La información tiene un papel relevante, tanto en la ciencia de la educación como en las TIC que permiten el acceso a la red de redes.

\section{§3. Propuesta Para un anÁlisis Filosófico-MetodolÓGico DE LA EDUCACIÓN MEDIADA POR LA TECNOLOGÍA}

Además de ser la educación una disciplina científica, la actividad educativa tiene interés estratégico en el desarrollo de la sociedad del conocimiento. Ambos aspectos requieren la atención de la filosofía de la ciencia ${ }^{12}$. La reflexión filosófico-metodológica sobre la ciencia de la educación mediada por

${ }^{12}$ En la sociedad del conocimiento, la educación ha adquirido una importancia estratégica por su capacidad para generar el llamado "capital intelectual". Gestionar adecuadamente el conocimiento es hoy condición indispensable para hacer posible la sostenibilidad del progreso, de modo que las pautas de actuación deben estar orientadas por criterios científicos. Pero además, en este contexto, lo que la sociedad demanda de la educación se ha desplazado desde la transmisión del conocimiento al desarrollo de procedimientos para gestionarlo. Se corre el riesgo de reducir los espacios educativos a espacios para el aprendizaje de gestión de la información. Abordar estas cuestiones es competencia de la filosofía de la ciencia, que tradicionalmente no ha tenido la educación entre sus centros de interés. 
la tecnología puede realizarse desde el ángulo de las ciencias de lo artificial (Simon, 1996; González, 2017). Esto comporta caracterizarla como ciencia aplicada. Confluyen así varios aspectos: $a$ ) La ciencia de la educación como ciencia de diseño ${ }^{13} ; b$ ) internet con una vertiente científica que sirve de base para su faceta tecnológica; y $c$ ) la inteligencia artificial, que tiene un papel relevante para diversos aspectos de la red de redes.

\subsection{Vertiente científica, faceta tecnológica y dimensión social}

Son ciencias de diseño tanto las ciencias de internet (web science, network science, etc.) como la inteligencia artificial. En esta vertiente científica también se sitúa la ciencia de la educación. Es importante este primer paso del análisis filosófico-metodológico, que consiste en la identificación del ámbito científico al que pertenecen las disciplinas mencionadas. Sucede, además, que cada una de ellas tiene relación con las tecnologías de la información y la comunicación.

Como segundo paso se requiere prestar atención a la esfera tecnológica. Por su incidencia en la ciencia de la educación y el desarrollo actual de la actividad educativa, hay que resaltar el llamativo crecimiento de las TIC. Han sido claves para la infraestructura tecnológica de internet, pero también para el desarrollo de la web y de las diversas aplicaciones prácticas (apps) de la última década. Las TIC constituyen un importante recurso tecnológico, que sirve como plataforma tecnológica donde tienen lugar muchas interacciones educativas, de modo que permite la realización de múltiples funciones.

${ }^{13}$ El uso del singular "ciencia de la educación" responde a la voluntad de destacar una disciplina autónoma sustantiva, que tiene por objeto de estudio el ámbito de realidad "educación". Se asume su diversificación en diferentes disciplinas, que se identifican con ella $a$ ) desde el punto de vista epistemológico, por utilizar la forma de conocimiento propia de la ciencia de la cual proceden, y $b$ ) ontológico, por el ámbito del conocimiento que les es propio: la pedagogía social, la organización escolar, la didáctica, la orientación y el diagnóstico, la teoría de la educación (pedagogía general). No se consideran ciencias de la educación otras ciencias sociales que se ocupan de los fenómenos educativos: $(i)$ cuando no los estudian como objeto específico (caso de la psicología de la educación, la sociología de la educación, la economía de la educación, etc.) y (ii) cuando sus disciplinas generadoras son diferentes a la ciencia de la educación, como la psicología, la sociología, la economía, etc. (Alonso, 2020: 171-172).

SCIO. Revista de Filosofía, n. ${ }^{\circ}$ 19, Noviembre de 2020, 229-259, ISSN: 1887-9853 
En cuanto al tercer paso, hay que atender la dimensión social que tiene tanto la red de redes como la inteligencia artificial. Ambas son mediadoras de las actividades educativas y contribuyen a la configuración filosófico-metodológica de la ciencia de la educación. La dimensión social se sitúa en la sociedad del conocimiento, donde destaca una morfología social novedosa, que se articula en redes que se potencian a través de intercambio de información. Estas redes son sistemas dinámicos y abiertos, donde los nodos pueden difundirse sin límites, mientras tengan la posibilidad de comunicarse entre sí.

La información es entonces la base "inmaterial", que permite la expansión de esta estructura social asentada en redes. En ella la morfología prevalece sobre la acción social y la determina. La correlación entre ambos aspectos ha configurado nuevos escenarios en los que se desarrolla la actividad humana: sociales, económicos, políticos..., pero también científicos y educativos. Así, el análisis filosófico-metodológico sobre la educación mediada por la tecnología atañe, fundamentalmente, a la educación electrónica en línea (on-line), pero también incluye al segundo tipo (la modalidad combinada o mixta).

El presente análisis se diversifica principalmente en tres ámbitos epistemológicos y metodológicos distintos, pero que son complementarios: la vertiente científica, la faceta tecnológica y la dimensión social. Su estudio requiere tener en cuenta tanto los niveles ontológicos: micro, meso y macro, como los marcos temporales: plazo inmediato, corto, medio, largo y muy largo (González, 2018). Queda fuera del ámbito propio de este artículo el realizar un análisis filosófico-metodológico sobre internet como plataforma tecnológica ${ }^{14}$.

\subsection{Incidencia de la red de redes y los agentes educativos}

Para el presente estudio, lo que interesa destacar es lo siguiente: $a$ ) la incidencia de la infraestructura de la red en el proceso de "cientificación" ex-

\footnotetext{
${ }^{14}$ El análisis, planteado desde una perspectiva interna, atendería a los factores clave de la transformación creativa de lo real, para dar lugar a algo tangible: $a$ ) el conocimiento, con sus diversas facetas -científico, específico tecnológico y evaluativo-; $b$ ) el quehacer en virtud del cual se realiza esa transformación creativa, para originar una realidad nueva; y $c$ ) el producto o artefacto, elaborado a partir del conocimiento disponible y el desarrollo del quehacer en el tiempo, siendo asumibles o razonables los costes (González, 2018: 78).
} 
perimentado por la ciencia de la educación en cuanto ciencia de diseño; $b$ ) su capacidad para ampliar el ámbito de la educación, haciendo posibles nuevos territorios temáticos (González, 2018: 84); y c) su inmenso potencial para promover nuevas prácticas y oportunidades de aprendizaje, configurando una educación sustancialmente diferente a la que hemos conocido.

Hoy las redes telemáticas se perfilan como las nuevas unidades básicas del sistema educativo: la información se "captura" y se difunde en cualquier lugar a través de la red, que es el espacio virtual donde se construye el conocimiento. Así, se desafía la identificación entre educación y escolarización. Esta situación fue anticipada hace una década por A. Collins y R. Halverson (2009: 1): "El mundo de la educación está experimentando actualmente una transformación masiva, como consecuencia de la revolución digital. Esta transformación es similar a la transición del aprendizaje a la escolarización universal que se produjo en el siglo XIX, como resultado de la revolución industrial. En la era del aprendizaje, la mayoría de lo que las personas aprendieron ocurrió fuera de la escuela. La escolarización universal llevó a la gente a identificar el aprendizaje con la escuela, pero ahora la identificación de los dos se está desmoronando".

La expansión de la educación mediada por la tecnología invita a pensar no solo en lo que es, sino también en lo que debería ser. Esto requiere poder anticipar el futuro posible, con el fin de establecer las pautas de actuación adecuadas para solucionar los problemas concretos que dificultan alcanzar los fines ${ }^{15}$.

Al reflexionar sobre lo que es la educación en línea, hay que partir de su configuración estructural, donde interviene la creatividad científica, pero en la que incide también la innovación tecnológica, que incorporan las TIC y en la que tiene también un papel cada vez más relevante la inteligencia artificial (González, 2017: 401-403). Esta configuración responde a una dinámica sometida a cambios en términos de historicidad (González, 2011), que tiene además una relación bidireccional con el entorno social, pues la educación está fuertemente entrelazada con un entramado de factores "externos".

\footnotetext{
${ }^{15}$ Algunas de esas dificultades están relacionadas, precisamente, con el difícil equilibrio entre pedagogía y tecnología.
} 
Entre estos factores cabe citar los estudiantes, las familias, la Administración, las disposiciones normativas (nacionales e internacionales), pero también las empresas y otros grupos $\mathrm{u}$ organizaciones, que tienen intereses diversos (económicos, sociales, culturales, etc.) en la educación. Por eso, la creatividad científica presente en la ciencia de la educación y las innovaciones que aportan las tecnologías mantienen una interacción dinámica, que plantea nuevos retos para la metodología de la investigación educativa y nuevas demandas al soporte tecnológico (más capacidad de almacenamiento, mejor accesibilidad, más velocidad, etc.), lo que incide en su progreso. Esto resulta central para abordar la complejidad, tanto estructural como dinámica, de la educación en línea.

El marco de análisis ha de atender a lo "interno" y lo "externo": (i) a la propia ciencia, mirando fundamentalmente a sus componentes epistemológicos y metodológicos; y (ii) como actividad sometida a constante cambio, que considera el papel relevante de los agentes educativos (alumnado, familias, profesorado, administración, Estados, organizaciones, etc.).

En su vertiente científica, la educación mediada por la tecnología se sitúa en el amplio ámbito de las ciencias de lo artificial (Simon, 1996: 3 y 4). Son ciencias aplicadas en cuanto afrontan procesos de indagación con el fin de dar solución a problemas concretos. Pertenecen al ámbito de lo artificial, que es resultado del desarrollo histórico de prácticas profesionales, que dan lugar a la "cientificación" de las reglas que orientan la resolución de problemas. Este resultado ya no responde a la intuición y la experiencia acumulada, sino a diseños científicos.

\subsection{Consolidación del carácter artificial de la educación como disciplina científica}

Como disciplina científica, la ciencia de la educación tiene puntos comunes con otras ciencias de la artificial que utilizan internet, como la economía, la documentación o la comunicación ${ }^{16}$. Respecto de etapas anteriores, la ac-

\footnotetext{
${ }^{16}$ Se trata de disciplinas que usan la red "para ampliar su campo de acción o crear novedades en sentido estricto" (González, 2018: 75).
} 
tual consolidación del carácter artificial de la educación como disciplina científica que trabaja a través de diseños ofrece importantes novedades:

1) Internet ha transformado la práctica del diseño, lo que ha facilitado su uso en el ámbito educativo. Inicialmente, el desarrollo de la ciencia de la educación ha estado vinculado a la "cientificación" de las habilidades profesionales (Niiniluoto, 1993: 11). Pero su despegue como ciencia de diseño efectiva se ha dado mucho más tarde, gracias al contexto vinculado al desarrollo de internet ${ }^{17}$.

Confluyeron al menos tres circunstancias: $a$ ) el desarrollo tecnológico de la red, $b$ ) la necesidad de vincular la investigación a la práctica, y c) la influencia, en el ámbito educativo, de los trabajos de Simon (en concreto, el libro The Sciences of the Artificial $)^{18}$. La relación entre la idea de una ciencia de diseño de la educación y la tecnología está presente ya en Allan Collins (1992: 15), cuando afirma: "La Tecnología nos proporciona unas herramientas poderosas para probar diferentes diseños, de manera que, en vez de Teorías de la educación, debemos empezar a desarrollar una ciencia de la educación"19.

2) En la educación del siglo XxI las direcciones científica y tecnológica son complementarias. Lo son en la práctica, pues hay una clara interacción dinámica entre ellas: la racionalidad científica y la racionalidad tecnológica se complementan ${ }^{20}$. Fueron los avances científicos los que impulsaron las

${ }^{17}$ De hecho, la idea de que la educación puede ser tratada como una ciencia de diseño se promovió en la década de los noventa, aunque hay algunos trabajos pioneros en la década anterior (Reif, 1978).

${ }^{18}$ La primera edición de The Sciences of the Artificial es del año 1969; fue actualizada en la segunda edicion en 1981 y, posteriormente, en la tercera edición de 1996, que alcanzaría una mayor difusión. El impacto de la aportación de Simon a las pretensiones científicas de la investigación educativa tiene un referente claro en la investigación basada en el diseño (design-based research), una orientación metodológica que se presenta como un medio para superar la brecha entre la teoría y la práctica. Tiene por objeto desarrollar una Ciencia de Diseño de la Educación que pueda orientar la creación y puesta en práctica de nuevos y más eficaces entornos de aprendizaje. Aunque los orígenes de esta línea metodológica en educación son anteriores, fue desde la publicación de los trabajos de Ann Brow (1992) y Allan Collins (1992), a comienzos de la década de los noventa, cuando su relación con las ciencias de diseño fue reconocida abiertamente.

${ }^{19}$ En el mismo sentido se expresaba ya F. Reif (1984: 2): "Personalmente creo que los desarrollos recientes en algunos campos científicos y tecnológicos hacen posible hoy progresos significativos hacia una Ciencia Aplicada efectiva de la Educación”.

${ }^{20}$ Hay que destacar también la presencia de la racionalidad económica, que tiene un papel determinante, en la orientación de la educación y también en el desarrollo de las tecnologías (González, 
innovaciones tecnológicas que permitieron el desarrollo de internet. Paralelamente, esas tecnologías, que en un principio no fueron creadas para la educación, han sido adaptadas para su uso en los entornos de enseñanza y aprendizaje. Esto ha transformado la acción pedagógica, en consonancia con las nuevas demandas sociales de un aprendizaje distinto y permanente (aprendizaje durante la vida).

3) Históricamente, la educación nunca impulsó el desarrollo de la tecnología, aunque los profesores siempre mostraron una especial habilidad para apropiarse de las invenciones útiles (así sucedió con herramientas hoy indisociables de la educación, como la escritura o el libro) ${ }^{21}$. Las adaptaron para su uso didáctico y las convirtieron posteriormente en recursos que son el verdadero motor de su desarrollo.

En las últimas décadas, las TIC suponen un desafío para la educación, si quiere liderar su uso y ponerlas al servicio de sus fines. A este respecto, una de las cuestiones fundamentales que atañen a la filosofía y metodología de la ciencia de la educación es si las innovaciones que han producido las muchas tecnologías introducidas en las aulas -y que fueron desarrolladas para otras actividades- están proporcionado conocimiento sistemático, de modo que permita orientar el avance de innovaciones futuras, de acuerdo con los fines que se consideran deseables para la educación.

La cuestión que se plantea es hasta qué punto las TIC en las aulas permiten realmente que los alumnos desarrollen sus conocimientos y capacidades personales. La ciencia de la educación debe ocuparse de los problemas concretos que surgen en relación con esta cuestión, por ejemplo: ¿qué innovaciones tecnológicas funcionan para los procesos educativos? y ¿en qué circunstancias son realmente eficientes? Como se trata de una ciencia aplicada de diseño, para afrontarlas, combina la predicción y la prescripción.

2018: 81). La racionalidad económica tiene un papel importante como mediadora entre la racionalidad científica y la racionalidad económica (González, 1998).

${ }^{21}$ La primera nació para el comercio, la segunda asociada a la religión. Tal vez la pizarra y la tiza fueron de las pocas herramientas inventadas específicamente para servir a la educación. Su homólogo moderno sería, entre todas las tecnologías digitales, la "pizarra digital" en el entorno de aprendizaje virtual (VLE por sus siglas en inglés). Laurillard (2012: 2). 


\section{§4. LA CIENCIA DE LA EDUCACIÓN COMO CIENCIA DE SÍNTESIS}

Aunque en cuanto ciencia de lo artificial, la ciencia de la educación es una ciencia de síntesis (Simon, 1996: 4 y 5), hay que tener presente que se trata de una ciencia aplicada que también puede analizar y, a partir del conocimiento que aporta, anticipar el futuro posible. Así, para solucionar problemas específicos, puede enunciar lo que se espera en un marco temporal (corto, medio o largo plazo) y, para dar una respuesta adecuada, proporcionar una serie de pautas que se concretan en un número finito de pasos.

La predicción es, pues, necesaria para el diseño científico, que se orienta hacia unos objetivos, antes de desarrollar unos procesos, en la búsqueda de unos resultados. Si desde el plano científico se quiere llegar al tecnológico, adaptado a la educación, entonces ha de haber una complementariedad entre el diseño científico y el diseño tecnológico. Por eso, si se quiere construir una teoría de diseño acerca de las innovaciones tecnológicas que son útiles para la educación, es necesario contar con una teoría subyacente, que permita interpretar los resultados.

En definitiva, para resolver los problemas de interés científico, se necesita anticipar y luego guiar la acción (González, 2018: 84). Además, hay que tener en cuenta que las ciencias de diseño, por una parte, son ciencias aplicadas, de modo que utilizan conocimiento teórico y lo amplían, pero construyen diseños para resolver problemas en lugar de explicaciones; y, por otra parte, ofrecen contenidos que enlazan con la aplicación de la ciencia en contextos que son variables (entornos educativos, sociales, culturales, económicos, etc.).

Los diseños son una expresión de síntesis. Dentro de la perspectiva interna -la vertiente científica-, los diseños indican los medios para conseguir los objetivos y comportan la presencia de criterios para medir los resultados que se obtienen. Así, están encaminados hacia ciertos objetivos, seleccionan los procesos y buscan los resultados. Cuando se ha llevado a cabo la tarea, los resultados que se obtienen muestran si el problema inicial está resuelto, si es preciso reformularlo o bien hay que descartarlo. Así es como estas ciencias buscan simultáneamente el desarrollo de la teoría y la innovación de la práctica.

Mediante la perspectiva externa se pone el foco en la dimensión social, que acompaña siempre a la actividad educativa en los niveles micro, meso y 
macro. La perspectiva interna se hace visible al hilo de la interdependencia entre predicción y prescripción, especialmente cuando -como sucede en educación- hay que afrontar problemas de complejidad, que tienen que abordarse mediante prescripciones. A su vez, la complejidad estructural y la complejidad dinámica de la actividad educativa no son ajenas a los factores sociales (culturales, políticos, económicos, etc.).

Metodológicamente, los procedimientos y métodos de la predicción pueden tener distintos niveles de fiabilidad. Pero la predicción puede considerarse como objetivo científico, una prueba para evaluar la validez de enunciados científicos y una guía de la actividad científica (González, 2010). Tras haber realizado una predicción, el acertar con la prescripción adecuada se vuelve más difícil, sobre todo cuando se trata de sistemas complejos como es el caso de los educativos. Esto es más problemático en la situacional actual, cuando entra en juego la complejidad dinámica de la tecnología.

Para prescribir es necesario saber cuál es la meta que se desea alcanzar. En su determinación intervienen los valores. Porque prescribir unas pautas de acción requiere una estimación acerca de lo que es conveniente y lo que es desaconsejable. Los valores internos de la prescripción resultan esenciales para el papel de la acción, pero son particularmente relevantes las valoraciones sociales. Por eso, no se puede desafiar a la tecnología para atender las necesidades de la educación, hasta que sepamos lo que queremos de ella.

Internet ha hecho evolucionar las pautas de comunicación, abriendo cauces nuevos descentralizados a la difusión de cualquier tipo de información, que compite con las instituciones escolares (Gimeno Sacristán, 2005: 60). La tecnología puede poner en riesgo la educación formal o bien convertirse en un instrumento valioso a su servicio. Pero precisar el papel que debe desempeñar la tecnología (el medio), requiere responder previamente a la pregunta acerca de qué es lo que se espera de la educación (el fin).

Las valoraciones sociales están también presentes en la deliberación acerca de los fines. El oportunismo tecnológico y, a veces, un entusiasmo tecnológico (Van de Poel, 2015) -bien intencionado, pero desinformado acerca de cualquier concepción teórica acerca de la enseñanza y el aprendizaje- pueden orientar la educación hacia fines no deseables, que comprometan el modelo de ser humano y de sociedad futuros. Además, hay que articular, de acuerdo 
con esos fines, lo que significa enseñar bien ${ }^{22}$, cuáles son los principios para diseñar una buena enseñanza y cómo esto permitirá a los estudiantes aprender.

La tecnología tiene mucho que ofrecer a la actividad educativa, pero la comunidad académica de la educación (que incluye a los docentes en cuanto investigadores) tiene que definir y defender el papel que le corresponde desempeñar. Hasta entonces, en esta ciencia de síntesis seguiremos corriendo el riesgo, como señala Laurillard (2012: 3), de ser dirigidos por la tecnología.

\section{§5. LOS DiSEÑOS PEDAGÓGICOS EN EL MARCO DE LAS CIENCIAS DE DISEÑO}

La ciencia de la educación utiliza conocimiento teórico y lo amplía para dar solución a problemas concretos. Al realizar los diseños educativos, se buscan los medios adecuados para alcanzar los objetivos propuestos, prestando atención también a los resultados que se obtienen. En esa empresa, el desarrollo de la teoría y la innovación de la práctica se implican simultáneamente.

Cabe ver los diseños pedagógicos como un emprendimiento científico que busca soluciones a los problemas, fundamentalmente vinculados al aprendizaje, que surgen en la sociedad interconectada. Se sitúan en el marco de las ciencias de diseño y conectan con las herramientas tecnológicas, que plantean nuevos retos para la enseñanza y abren posibilidades para explorar diferentes aspectos del proceso de aprendizaje y para mejorar su conocimiento.

Pero además de estar en el punto de partida desde el punto de vista epistemológico, el diseño está en el punto de llegada desde una perspectiva metodológica. En este sentido, permite evaluar los usos de la tecnología, para comprobar si son efectivos. Puede crear ambientes de aprendizaje novedosos, para identificar las variables que influyen en el éxito o el fracaso de las innovaciones, con la mirada puesta en cómo se adecuan a los objetivos de la educación.

${ }^{22}$ En la actualidad, el centro de atención de la educación se está desplazando de la enseñanza al aprendizaje, pero este debate queda fuera del ámbito de este trabajo.

SCIO. Revista de Filosofia, n. ${ }^{\circ}$ 19, Noviembre de 2020, 229-259, ISSN: 1887-9853 
El interés por el diseño para abordar problemas educativos venía siendo anecdótico y estaba vinculado a ámbitos de investigación muy específicos ${ }^{23}$. $\mathrm{Su}$ ampliación gradual y la relevancia que está adquiriendo, especialmente en la última década, tiene una clara relación con la normalización del uso de internet ${ }^{24}$.

Habitualmente, los diseños educativos se realizan para orientar la búsqueda de soluciones prácticas mediante intervenciones educativas. Estas intervenciones -aplicaciones de la ciencia- contribuyen a mejorar la manera de entender la ciencia aplicada: la indagación acerca de los procesos de enseñanza-aprendizaje permite pensar en soluciones. Así se elabora la teoría, destinada a orientar procesos más efectivos que mejoren la práctica. Tal indagación no puede ser puramente interna, por eso ha de incorporar los cambios del entorno donde se desarrolla el aprendizaje.

Los diseños educativos pueden implementarse en cualquier modalidad educativa: (i) la presencial, donde los cambios que se promueven afectan al ambiente y la cultura del aula; (ii) la semipresencial, que combina trabajo en el aula y fuera de ella, y (iii) la educación a distancia en línea. En cada una de ellas cabe prescribir reglas de acción que cumplan los requisitos de ciencia. Sin embargo, donde el diseño puede optimizar el uso adecuado de la tecnología es en la educación en línea, ya que permite el máximo aprovechamiento pedagógico de los recursos multimedia que ofrecen las tecnologías ${ }^{25}$.

El éxito del diseño instruccional está condicionado por la restricción en la amplitud del campo educativo abordado. Tiene mayor relevancia en la modalidad de enseñanza en línea, puesto que caracteriza de manera concreta los distintos elementos que configuran las acciones formativas que se llevan a cabo en los entornos virtuales. Hay un proceso sistemático que no deja lugar

${ }^{23}$ Como se señaló, aunque sus antecedentes son algo anteriores, "investigación de diseño" aparece en la investigación educativa a comienzos de la década de los noventa del pasado siglo.

${ }^{24}$ Internet hace posible diferentes formas de trabajo, que la han ido configurando como una herramienta eficaz para el diseño, lo que permitió ampliar su uso a nuevos escenarios de actuación.

${ }^{25}$ Las TIC mejoran considerablemente las capacidades de computación. Esto permite superar ciertas limitaciones del procesamiento de la información por los agentes y propicia un mejor manejo de algunas variables acerca de la realidad. Por otra parte, procede también señalar que las herramientas tecnológicas se ponen, en principio, al servicio de los objetivos de la educación. Pero internet, como plataforma tecnológica, determina la formulación operativa. 
para la improvisación o para cualquier pauta no planificada: I) Se establecen las metas (objetivos) de la enseñanza de acuerdo con las necesidades detectadas; II) se desarrollan las estrategias, actividades y recursos que hacen posible alcanzarlas en un número finito de pasos (proceso); y III) se fijan los procedimientos para la evaluación de los resultados del aprendizaje y de toda la instrucción.

Además, la modalidad de enseñanza en línea se adapta bien a las peculiaridades de la inteligencia artificial ${ }^{26}$, que es una inteligencia básicamente computacional: diseña algoritmos para diseñar otros algoritmos de acciones, dirigidos a resolver tareas que están bien especificadas. Asimismo, el diseño es intrínsecamente computacional, en cuanto consiste en procesar las implicaciones de los supuestos iniciales y sus combinaciones. Diseñar, como lo entendió Simon (2007: 151), es hacer acopio de información sobre lo que se sigue de lo que uno ha propuesto.

Para caracterizar el diseño instruccional en la formación virtual, Coll, Mauri y Onrubia (2008) proponen hablar de "diseño tecnoinstruccional o tecnopedagógico". Consideran que en él confluyen de forma indisociable dos dimensiones: primero, hay una dimensión tecnológica, relacionada con la selección de las herramientas tecnológicas que se consideran adecuadas al proceso formativo que se desea realizar (plataforma virtual, aplicaciones de software, recursos multimedia, etc.), teniendo en cuenta las posibilidades existentes y sus limitaciones. Segundo, posee una dimensión pedagógica, que aporta el conocimiento de las características de los destinatarios, los objetivos y/o competencias, los contenidos y la planificación de las actividades (que incluyen orientaciones y sugerencias sobre el uso de las herramientas tecnológicas para su desarrollo). También incluye un plan de evaluación de los procesos y de los resultados.

${ }^{26}$ La inteligencia artificial puede ubicarse también en el ámbito temático de las ciencias de lo artificial, puesto que tiene los rasgos que caracterizan a estas ciencias: es una ciencia de síntesis, versa acerca de algo construido por los humanos, para ampliar sus posibilidades humanas, y está orientada a nuevos objetivos, procesos y resultados. 


\section{§6. El CARÁCter limitAdo DEL DiSEÑo PARA ABARCAR EL CONJUNTO DE LA EDUCACIÓN}

El desarrollo de los diseños pedagógicos (o tecnopedagógicos) puede analizarse siguiendo el enfoque filosófico-metodológico propio de las ciencias de lo artificial. El foco de atención puede estar en la influencia de internet en esos diseños, estudiando cómo afecta a la formación de los objetivos, de los procesos y de los resultados. Para ello hay que tener en cuenta factores "internos" y "externos", que tienen importantes consecuencias para la educación y, por ende, para las oportunidades de las personas. Porque los objetivos, procesos y resultados de estos diseños se incardinan a lo largo de dos líneas principales:

- La motivada por la preocupación "interna" se ocupa del logro de los objetivos de la educación vía internet, considerados por sí mismos; esto es, analizados independientemente del entorno. Son los aspectos que tienen relación con el propio desarrollo de la enseñanza en línea: accesibilidad, velocidad, etc.

- La orientada a lo "externo", es decir, al ajuste entre los distintos elementos del currículum y los factores exógenos (sociales, culturales, económicos, políticos, etc.). Porque sus demandas modulan en gran medida los objetivos y, además, interactúan con el logro de estos objetivos de diversas maneras ${ }^{27}$.

Los factores internos están relacionados con los elementos constitutivos de las ciencias: lenguaje, estructura, conocimiento, métodos, actividad, fines y valores (González, 1997 y 2012). Son la base que, al hacer los diseños educativos, da soporte a la interacción entre dos tipos de disciplinas de lo artificial: por un lado, las ciencias de internet y las aportaciones de la inteligencia artificial; y, por otro lado, la ciencia de la educación (y el conjunto de las disciplinas pedagógicas en las que se diversifica).

Todas estas disciplinas son expresión de las ciencias del diseño, puesto que son ciencias de lo artificial y se modulan como ciencias de síntesis, aunque tengan elementos de análisis. Con sus aportaciones, confluyen en la empresa

${ }^{27}$ Esto, a mi juicio, adquiere una dimensión mayor en los diseños en red. 
humana de hacer diseños orientados a objetivos específicos, que llevan a seleccionar procesos, en la búsqueda de resultados, para ampliar las posibilidades humanas. En este caso, mejorando los aprendizajes para potenciar las capacidades que van a permitir elegir el tipo de vida que se quiere vivir. Como consecuencia de su desarrollo interno y de la influencia de los factores externos, la dinámica de esa empresa científica cambia con el tiempo, lo que es expresión de su historicidad.

Para resolver los problemas de aprendizaje que surgen en el nuevo contexto, la dinámica científica desarrolla diseños. Se hacen para probar las soluciones -nuevos escenarios, recursos, materiales, etc.- y se contrastan empíricamente, para observar si funcionan en el contexto al que se aplican, formulando preguntas sobre sus usos efectivos ${ }^{28}$. Las herramientas tecnológicas, además de plantear nuevos retos para la enseñanza, abren posibilidades para explorar diferentes aspectos del proceso de aprendizaje y mejorar su conocimiento. Proporcionan también nuevas herramientas para el diseño, nuevas formas de trabajo y abren escenarios de actuación novedosos en el ámbito educativo.

Al formular los objetivos, hay que atender a todas las variables que entran en juego, dentro de las limitaciones del entorno -que en la educación en línea serán distintas a las de la investigación que se realiza en el aula- y pueden afectar al resultado. Al seleccionar los procesos (por ejemplo, para probar hipótesis específicas sobre preguntas acerca de determinados usos efectivos de la tecnología), las pautas de actuación que se prescriban han de llevar a actividades que permitan alcanzar los objetivos.

Esto va a permitir identificar y corregir el error en el momento en el que se produzca, para así reorientar cualquier fase del desarrollo del diseño, sin esperar al final del proceso. Es importante analizar por qué no está funcionando un diseño y corregir aquello que apunta a las razones del fracaso, ya que la información que se obtiene es relevante. Pero es al evaluar los resultados, en función del éxito o el fracaso de la intervención, cuando se mide la adecuación del diseño.

${ }^{28}$ Los docentes asumen el papel de investigadores cuando realizan o prueban sus diseños o de coinvestigadores cuando adaptan los diseños, los mejoran y evalúan los efectos de los diferentes aspectos del experimento. 
El diseño instruccional (instructional design) permite el máximo aprovechamiento pedagógico de la incorporación de las tecnologías educativas. $\mathrm{Al}$ vincular las teorías del aprendizaje a la práctica educativa, proporciona además una guía para una planificación sistemática y rigurosa de la acción pedagógica, que ya no se fundamenta en la experiencia acumulada por el profesor (lo que sería aún una "técnica", pero no una "ciencia")" 29 . Así, el diseño instruccional, además de contribuir a la mejora de la práctica, busca ofrecer conocimiento fiable para las intervenciones futuras.

M. David Merril (2009) propuso cinco principios instruccionales prescriptivos, que considera fundamentales para mejorar la calidad de la enseñanza en cualquier situación: (i) la centralidad de la tarea, (ii) la activación, (iii) la demostración, (iv) la aplicación y (v) la integración. Charles Reigeluth (2012) considera que estos principios ofrecen un buen resumen, aunque sea general, de las características más importantes para una instrucción de alta calidad.

El principio de centralidad de la tarea (Merril, 2009) establece que la instrucción debe emplear una estrategia de enseñanza centrada en la tarea y debe realizarse mediante una progresión de tareas completas cada vez más complejas. Las tareas son propuestas didácticas que tienen como objetivo la integración del saber y del saber hacer, movilizando todos los recursos disponibles de la persona y permitiendo la transferencia de saberes a la vida cotidiana.

${ }^{29}$ A lo largo del siglo xx, los modelos de instrucción tuvieron su fundamento en las teorías del aprendizaje predominantes en cada momento: el conductismo en los sesenta, la teoría de sistemas en los setenta, la teoría cognitiva en los ochenta, el constructivismo y la teoría de sistemas en los noventa. En la actualidad, para ofrecer una guía para el diseño, se desarrollan teorías instruccionales bien contrastadas, tanto respecto de la tarea como para el espacio de instrucción, que combinan lo mejor de las teorías y modelos conductistas, cognitivistas y constructivistas. También hay que añadir los modelos de instrucción basados en el conectivismo, que es la concepción del aprendizaje más influyente entre las que surgieron en el nuevo siglo como consecuencia del uso de la Tecnología y su influencia en el aprendizaje (Benítez, 2010)

Anderson y Dron (2012: 150 y 151) han observado una relación entre las teorías del aprendizaje sobre las que se construyeron los modelos de diseño instruccional y el desarrollo tecnológico. Así, en la etapa previa a internet, los modelos pedagógicos de carácter cognitivo conductual surgieron en un entorno tecnológico que limitaba la comunicación a modos "uno a uno" y "uno a muchos". El constructivismo social floreció en la web 1.0, en un contexto en que ya era posible la comunicación "muchos-amuchos". El conectivismo ha tenido un creciente protagonismo con el aprendizaje de máquina (machine learning) y el mundo de redes con web 2.0. Con respecto a lo que puede traer el siguiente paso en la evolución tecnológica, algunos consideran que la web 3.0 es la red semántica. 
Contienen actividades cuyo objetivo es el dominio de una habilidad o un procedimiento concreto o la comprensión de conceptos. A su vez, las actividades se componen de secuencias de ejercicios que buscan la adquisición de una habilidad o un procedimiento concreto y sencillo. Así secuenciada, una tarea compleja podría ser resuelta apelando a una inteligencia computacional entendida, en el sentido de Giusseppe Primiero (2007: 521), como la ejecución de cualquier acción que puede ser descrita en términos de instrucciones y que se puede ver para resolver tareas. Este es -a mi juicio- el ámbito en el que son comparables la inteligencia humana y la inteligencia artificial.

\section{§7. LOS LÍMITES DE LA INTELIGENCIA COMPUTACIONAL}

En el rango de la inteligencia computacional está, según Giusseppe Primiero, cualquier forma de comportamiento inteligente que pueda ser producida por la ejecución de algoritmos bien definidos, aunque sean complejos (2007: 524). Cree, sin embargo, que el principio fundamental de la verdadera inteligencia artificial general, pensada como habilidad para actuar en cualquier condición inespecífica con el espíritu inteligente que un humano podría mostrar, está todavía muy lejos (Primiero, 2007: 522).

Su definición de inteligencia computacional incluye todo lo que las máquinas, en cuanto artefactos tecnológicos -y no meramente como abstracciones matemáticas-, ya son capaces de hacer: por ejemplo, conducir un coche, diagnosticar condiciones médicas y proponer una cura adecuada. Excluye entonces todo lo que no es, por ahora, totalmente definible de manera algorítmica, como tomar una decisión moral. A este respecto, sin entrar en el debate acerca de si seguir una regla requiere o no inteligencia, la tarea así planteada nos sitúa ante habilidades de carácter mecánico, no estrictamente intelectuales que son propias de la inteligencia computacional y que podrían estar al alcance de una máquina, que además tiene una capacidad de computación mayor ${ }^{30}$.

\footnotetext{
${ }^{30}$ Para la realización de las tareas que se llevan a cabo de forma colaborativa en el aula, entran en juego la percepción -conocimiento-, la volición y los valores, que son los tres dominios principales que Wenceslao J. González (2017: 404) identifica en la inteligencia humana. A mi juicio, la movilización de estos tres dominios está motivado por la forma de interacción cara a cara en el aula. El diseño instruc-
} 
González (2017) plantea la inteligencia humana y la inteligencia artificial en un contexto de fondo, que lleva a distinguir entre "inteligencia" y "racionalidad", además de diferenciar entre "inteligencia humana" e "inteligencia computacional". Así, la concepción meramente instrumental propia del proceso en IA nos sitúa ante un tipo de racionalidad-de índole procesual-más que ante una inteligencia real o verdadera, si entendemos "inteligencia" de acuerdo con su significado etimológico de intus-legere, es decir, como un acto intelectual directo para captar algo formal o empírico sin mediación. Esto supone que la inteligencia computacional es entonces un procesamiento operativo, de modo que comúnmente se mide en términos cuantitativos. Se trata fundamentalmente de un proceso que está orientado hacia objetivos dados.

Enumera una serie de rasgos por los que la inteligencia humana se distingue de la inteligencia computacional. Tiene una dimensión más amplia y un alcance mayor, así como una configuración más compleja. 1) Mediante la inteligencia humana se reconoce la complejidad estructural y dinámica. 2) La inteligencia humana no es meramente operativa o puramente funcional. Así, no es simplemente una racionalidad de medios. 3) La inteligencia humana puede captar los contenidos de las humanidades, la ciencia y la tecnología, porque puede hacer frente a los diferentes niveles de análisis (como universal y particular, concreto y abstracto, etc.). 4) La inteligencia humana no está limitada a la esfera cognitiva, sino que atiende también a las acciones -incluida su motivación en términos de afectos, sentimientos o emociones-con sus voliciones y a los valores.

El análisis de Wenceslao J. González (2017) apunta a una serie de cuestiones de especial interés para el uso educativo de la tecnología, que trasciende el ámbito de este trabajo. Me gustaría adelantar, sin embargo, una serie de consideraciones sobre las que, a mi juicio, cabe reflexionar dentro del presente terreno de análisis filosófico-metodológico:

(i) El diseño pedagógico en la educación en línea es un diseño instruccional y depende de la creatividad de los diseños científicos y el apoyo de innovaciones tecnológicas. (ii) Esta interacción proporciona el marco para tipo de interacción es diferente en la educación en línea, donde prevalece el plano cognitivo. 
la consolidación científica de la educación dentro de las ciencias de lo artificial. (iii) La inteligencia humana tiene, entre otras facetas, una dimensión computacional; la inteligencia artificial es computacional; y el diseño es, en principio, computacional. (iv) La inteligencia humana no puede reducirse a su dimensión computacional. Pero el diseño instruccional presenta limitaciones a la hora de abordar otras dimensiones, puesto que determinados procesos no se pueden operativizar ${ }^{31}$. Así pues, $(v)$ el diseño instruccional presenta limitaciones, para atender al desarrollo de capacidades necesarias para la resolución de problemas, el pensamiento crítico, la iniciativa o la creatividad; para atender, en definitiva, a la totalidad de la educación ${ }^{32}$. (vi) Instrucción y educación no son términos equivalentes. La instrucción (ins-struere: "construir en o sobre") hace referencia a procesos de transmisión de información planificada (adquirir unos conocimientos concretos) y se relaciona con el significado de $\underline{e}$ dŭcāre, que remite a la asimilación cognitiva de información por parte del estudiante. Pero no incluye la dimensión que le otorga el significado de $\bar{e}$ dŭcēre ("sacar afuera"), relacionado con el desarrollo de las potencialidades humanas, que es el fin de la educación.

Los límites del diseño se aprecian en las dificultades para abarcar la totalidad de la educación, que es un proceso más complejo que la instrucción, porque esta se limita a transmitir destrezas, técnicas o conocimientos, criterios descriptivos o prescriptivos. Lo que la educación transmite es lo que se considera valioso de la cultura (el patrimonio cultural), para conseguir la integración y el progreso social. Su objetivo es capacitar a las personas para actuar de modo intencional, de acuerdo con ese conocimiento adquirido y la experiencia acumulada frente a situaciones nuevas. El fin de la educación es el pleno desarrollo de la personalidad humana, de modo que implica todo

${ }^{31}$ También Reigeluth (2012) señala dificultades para atender al desarrollo social y emocional, actitudes, valores... Sin embargo, ha desarrollado estrategias de enseñanza en relación con distintos tipos de aprendizaje de carácter cognitivo (por ejemplo, división en partes, repetición, inducción y mnemotecnia para la memorización; analogías, organizadores previos, comparaciones, contraste... para la comprensión conceptual, etc.).

${ }^{32}$ Se trata de capacidades constitutivas de los fines de la educación. Sin embargo, ha mostrado su eficacia para el desarrollo de determinadas aptitudes que pueden ser compatibles con los fines estrictos del progreso económico. 
aquello que contribuye a desarrollar las habilidades, aptitudes y posibilidades del individuo y a crear, corregir y ordenar sus ideas, hábitos y tendencias.

El acto educativo es, pues, más complejo y la educación no puede reducirse a la instrucción. Tampoco se puede abordar sin atender a su complejidad, tanto estructural (requiere analizar los elementos que la componen) como dinámica (en cuanto proceso objetivo, que contribuye de manera específica a la integración entre la teoría y la práctica a través del tiempo). Abordar la educación requiere, pues, contemplarla en esta doble dimensión: como objeto de conocimiento y como factor de cambio por parte de sus agentes, por lo que se constituye en un elemento integrador y coherente entre la teoría y la práctica de la enseñanza.

Ese es el sentido de la educación, en tanto disciplina científica. El análisis filosófico-metodológico de la ciencia de la educación como ciencia de diseño ha revelado la relación del diseño con la tecnología y su potencial para cambiar la educación. Poner la tecnología al servicio de la educación requiere al menos dos aspectos: 1) una deliberación racional acerca de los fines y 2) utilizar el conocimiento que la ciencia actual nos proporciona acerca del aprendizaje, para identificar lo que la educación necesita de la tecnología para alcanzar esos fines.

\section{REFERENCIAS BIBLIOGRÁFICAS}

Alonso, A. M. (2020). La Ciencia de la Educación ante las diversas opciones metodológicas de índole disciplinar. ArtefaCTOS. Revista de estudios de la Ciencia y la Tecnología 9(1), 175-198. doi: https://dx.doi.org/10.14201/ art202091175198.

Anderson, T., Dron, J. (2011). Tecnología para el aprendizaje a través de tres generaciones de pedagogía a distancia mediada por tecnología, consultado el 06/05/2018. Revista mexicana de Bachillerato a distancia 3(6), 136-154. Disponible en: $<$ http://revistas.unam.mx/index.php/rmbd/article/ view/65057>.

Benítez, M. G. (2010). El Modelo de diseño instruccional ASSURE aplicado a la educación a distancia, consultado el 02/04/2019. Tlatemoani. Revista 
Académica de Investigación (1), 1-15. Disponible en: < http://www.eumed. net/rev/tlatemoani/01/pdf/63-77_mgbl.pdf $>$.

Brown, A. L. (1992). Design Experiments: Theoretical and Methodological Challenges in Creating Complex Interventions in Classroom Settings. The Journal of the Learning Sciences 2(2), 141-178.

Cabero, J. (2001). Tecnología educativa. Diseño y utilización de medios en la enseñanza. Barcelona: Paidós.

Childers, G., Gail Jones, M. (2015). Students as Virtual Scientists: An exploration of students' and teachers' perceived realness of a remote electron microscopy investigation, consultado el 09/02/2019. International Journal of Science Education 37(15), 2433-2452. doi: https://doi.org/10.1080/ 09500693.2015.1082043.

Coll, C. Mauri, T., Onrubia, J. (2008). Los entornos virtuales de aprendizaje basados en el análisis de casos y la resolución de problemas. En C. Coll y C. Monereo (eds.), Psicología de la educación virtual (pp. 213-232). Madrid: Morata.

Collins, A. (1992). Toward a Design Science of Education. En E. Scanlon y T. O'Shea (eds.): New Directions in Educational Technology (pp. 15-23). Berlin/Heidelberg: Springer/Verlag.

Collins, A., Halverson, R. (2009). Rethinking Education in the Age of Technology: The Digital Revolution and the Schools. New York, NY: Teachers College Press.

Gimeno Sacristán, J. (2005). La educación que aún es posible. Madrid: Morata.

Gonzalez, W. J. (1997). Progreso científico e innovación tecnológica: La 'Tecnociencia' y el problema de las relaciones entre Filosofía de la Ciencia y Filosofía de la Tecnología. Arbor 157(620), 261-283.

Gonzalez, W. J. (2010). Racionalidad científica y racionalidad tecnológica: La mediación de la racionalidad económica. Ágora, Papeles de Filosofía 17(2), 95-115.

González, W. J. (2010). La predicción cientifica: Concepciones filosófico-metodológicas desde H. Reichenbach a N. Rescher. Barcelona: Montesinos. 
González, W. J. (2011). Conceptual Changes and Scientific Diversity: The Role of Historicity. En W. J. González (ed.), Conceptual Revolutions: From Cognitive Science to Medicine (pp. 39-62). A Coruña: Netbiblo.

González, W. J. (2012). La Economía en cuanto Ciencia: Enfoque desde la complejidad, consultado el 09/12/2018. Revista Galega de Economía 21(1), 1-30. Disponible en: <http://www.usc.es/econo/RGE/Vol21_1/castelan/ bt1c.pdf $>$.

González, W. J. (2015). Philosophico-Methodological Analysis of Prediction and its Role in Economics. Dordrecht: Springer.

González, W. J. (2017). From Intelligence to Rationality of Minds and Machines in Contemporary Society: The Sciences of Design and the Role of Information, consultado el 06/10/2017. Minds and Machines 27(3), 397-424. doi: 10.1007/s11023-017-9439-0. Disponible en: <https://link.springer. com/article/10.1007/s11023-017-9439-0>.

González, W. J. (2018). Internet en su vertiente científica: Predicción y prescripción ante la complejidad, consultado el 28/04/2018. ArtefaCToS. Revista de estudios de la Ciencia y la Tecnología 7(1), 75-97. doi: http:// dx.doi.org/10.14201/art2018717597.

González, W. J. (2019). Internet y Economía: Una relación multivariada en el contexto de complejidad. Energeia: Revista internacional de Filosofía y Epistemología de las Ciencias Económicas 6(6), 11-36.

Gunawardena, C. N., McIsaac, M. S. (1996). Distance Education. En D. H. Jonassen, Handbook on Research for Educational Communications and Technology (pp. 407-437). New York, NY: Macmillan.

Laurillard, D. (2012). Teaching as a Design Science: Building Pedagogical Patterns for Learning and Technology. New York, NY: Routledge.

Laurillard, D. (2007). Technology, Pedagogy and Education: concluding comments. Technology, Pedagogy and Education 16(3), 357-360. doi: https:// doi.org/10.1080/14759390701614496.

Niiniluoto, I. (1993). The Aim and Structure of Applied Research. Erkenntnis $38(1), 1-21$. 
Merrill, M. D. (2009). First principles of instruction. En C. M. Reigeluth y A. A. Carr-Chellman (eds.), Instructional-design theories and models: Building a common knowledge base, v. 3 (pp. 41-56). New York, NY: Routledge.

Primiero, G. (2007). Algorithmic Iteration for Computational Intelligence. Minds and Machines, 27(3), 521-543. Disponible en: <http://eprints.mdx. ac.uk/21262/>.

Reif, F. (1978). Toward an Applied Science of Education: Some key questions and directions. Instructional Science (7), 1-14.

Reigeluth, Ch. (2012). Teoría instruccional y tecnología para el nuevo paradigma de la educación, consultado en 04/03/2018. RED, Revista de Educación a Distancia (32), 1-22. Disponible en: <http://www.um.es/ead/ $\mathrm{red} / 32>$.

Simon, H. A. (1996). The Sciences of the Artificial, 3. ${ }^{\mathrm{a}}$ ed. Cambridge, MA: The MIT Press.

Simon, H. A. (2007). Formación de problemas, detección de problemas y solución de problemas de diseño. En W. J. González (ed.), Las Ciencias de Diseño: Racionalidad limitada, Predicción y Prescripción (pp. 149-159). A Coruña: Netbiblo.

Tiropanis, T., Hall, W., Crowcroft, J., Contractor, N., Tassiulas, L. (2015). Network Science, Web Science, and Internet Science. Communications of ACM 58(8), 76-82.

Van de Poel, I. (2015), Values in Engineering and Technology. En W. J. González (ed.), New Perspectives on Technology, Values, and Ethics (pp. 29-46). Dordrecht: Springer.

Williams, P., Schrum, L., Sangra, A., Guardia, L (2003). Modelos de diseño instruccional. Fundamentos del diseño técnico-pedagógico en e-learning, consultado el 09/04/2016. Cataluña: Universitat Oberta de Cataluña. Disponible en: $<$ https://cutt.ly/Xhkb4J7>. 